\title{
UCRL-TR-219363
}

LAW RENCE LIVERMORE N A T IO N A L LABORATORY
Transition-ready technologies and expertise from the Chemical and Biological National Security Program at LLNL

Peggy A. Folta, Mary T. McBride

February 28, 2006 
This document was prepared as an account of work sponsored by an agency of the United States Government. Neither the United States Government nor the University of California nor any of their employees, makes any warranty, express or implied, or assumes any legal liability or responsibility for the accuracy, completeness, or usefulness of any information, apparatus, product, or process disclosed, or represents that its use would not infringe privately owned rights. Reference herein to any specific commercial product, process, or service by trade name, trademark, manufacturer, or otherwise, does not necessarily constitute or imply its endorsement, recommendation, or favoring by the United States Government or the University of California. The views and opinions of authors expressed herein do not necessarily state or reflect those of the United States Government or the University of California, and shall not be used for advertising or product endorsement purposes.

This work was performed under the auspices of the U.S. Department of Energy by University of California, Lawrence Livermore National Laboratory under Contract W-7405-Eng-48. 


\section{Transition-ready technologies and expertise from the Chemical and Biological National Security Program at LLNL Wednesday, February 22, 2006}

HSARPA has initiated a new Bioinformatics and Assay Development solicitation, BIAD2 (BAA 06-01), to address a number of technology gaps and requirements for biodetection (www.hsarpabaa.com). This solicitation will leverage the vast research and development capabilities of the private sector and academia in order to meet the needs of HSARPA and Homeland Security. In order to meet these requirements, this solicitation will:

- Develop and validate actionable assays for the public and private sector

- Develop and validate new assays and novel assay methodologies to enhance existing detection systems and enable future detection platforms

- Develop next generation assays which are robust against novel, emerging and engineered threats

- Develop novel assays that detect low levels of ribonucleic acid (RNA)-based viral threats in complex backgrounds

- Develop novel assays to characterize the viability, degree of virulence or toxicity, and countermeasure resistance of a biological agent

- Develop new bioinformatics tools to support assay development and assay validation

The Lawrence Livermore National Laboratory (LLNL) Bioassays and Signature Program (BSP) develops nationally-validated detection and identification assays to cover the full range of biological threat agents, starting from human, animal, and plant pathogens on the Select Agent list. The assays that have been co-developed by the CDC and the BSP are used internationally and represent the gold standard for molecular detection of select agent pathogens for the public health community. They are also used in the DHS environmental monitoring operations such as BioWatch and DHS National Security Special Events support. These reagents have been used to process and analyze more than 5 million samples and have delivered exceptional performance for the end users, with zero false positives since their deployment. Currently, highly-multiplexed nucleic acid assays that represent the "next-generation" in BioWatch assays have been developed at LLNL and are in final evaluation at CDC. LLNL is a full partner to the LRN network, as well as a formal LRN member, sanctioned to perform these diagnostics on field samples.

The Program has assay extensive experience developing, testing, validating, deploying, and commercializing biodetection assays and instrumentation. The following pages contain additional descriptions of our Program's capabilities. We have a demonstrated track record of established collaborations and look forward to partnering with offerers to the HSARPA BAA to continue to meet the national needs and requirements.

For additional information, please contact Mary McBride at: mcbride2@1lnl.gov. 


\section{The Chemical and Biological National Security Program at LLNL}

The mission of the Chemical and Biological National Security Program, (CBNP) is to develop and field advanced strategies that dramatically improve the nation's capabilities to prevent, prepare for, detect, and respond to terrorist use of chemical or biological warfare agents. The science, technology and integrated systems we provide are informed by and developed with key partners and end-users. The key science and technology attributes of the program are centered around informatics, assays, and instrumentation for environmental and agricultural targets; biological knowledge discovery from disparate data sources; and a variety of response and operations efforts centered around forensics, restoration, and atmospheric modeling. CBNP provides integrated access to a broad range of Laboratory skills and facilities, including:

- Special facilities in \$5B infrastructure

○ BSL-3 Pathogen Laboratory (activation Spring 2006)

- Fermentation Laboratory

- Forensic Science Center

- National Atmospheric Release Advisory Center (24x7)

- Biosecurity and Nanoscience Support Laboratory

- Micro Technology Center

- Center for Accelerator Mass Spectrometry

- Over 180 CBNP staff

- DHS Biodefense Knowledge Center (24x7)

The following information covers the specific capabilities and expertise:

- Bioassays and Signatures Program:

- Assay Development Pipeline: An Overview

- Sequencing Capabilities

- BioInformatics

- DNA Signature Development

- Protein Signature Development

- Broad Applications

- TaqMan Nucleic Acid Assay Development

- Multiplexed Nucleic Acid Assay Development

- Dissemination of Assay Products into User Communities

- Multiplexed Immunoassay Development

- Development of Protein Based Detection Reagents

- Functional Assays and Signatures

- High Throughput Laboratory

- BioInstrumentation Development Program

- A Multiplexed Diagnostic Platform for use at Point-of-Care

- An Advancement in Detection Systems - BioBriefcase

- High Throughput Rapid Viability Analysis 


\section{Chemical and Biological National Security Organization}

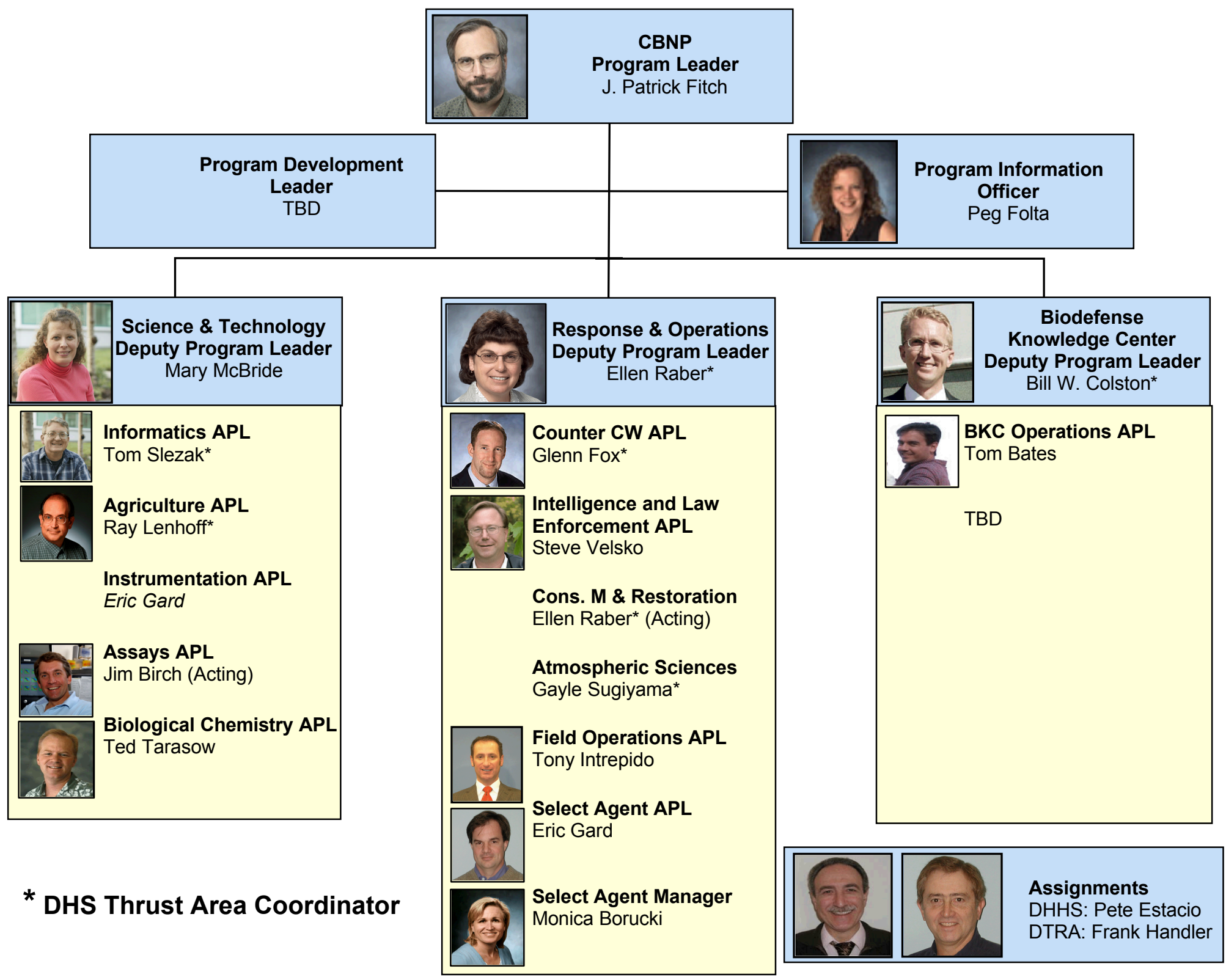




\section{Bioassays and Signatures Program: Assay Development Pipeline}

The Bioassays and Signatures Program (BSP) at Lawrence Livermore National Laboratory (LLNL) have constructed a robust technical architecture (Figure 1) for the rapid development of highest-quality nucleic acid assays, tailored to end-users specifications. This process is generic enough to be used for any signature type and any organism. Our pipeline process has been used to provide high-confidence detection assays for many priority bioterrorism agents. The pipeline process begins with an analysis of all available genomic sequence information, which forms the basis for the development of signatures. A signature is a region or set of regions on a chromosome that is unique to that organism. The pipeline process of signature discovery and assay development utilizes a whole-genome approach where the entire genome is computationally examined to identify candidate regions that might be unique to the property or characteristic of interest. Knowledge about the function of the gene is not required, and many thousands of possible signatures can be rapidly generated to identify a few hundred of the most promising. Candidate signatures can be selected based on performance criteria for specific detection technologies. Our nucleic acid assays employ

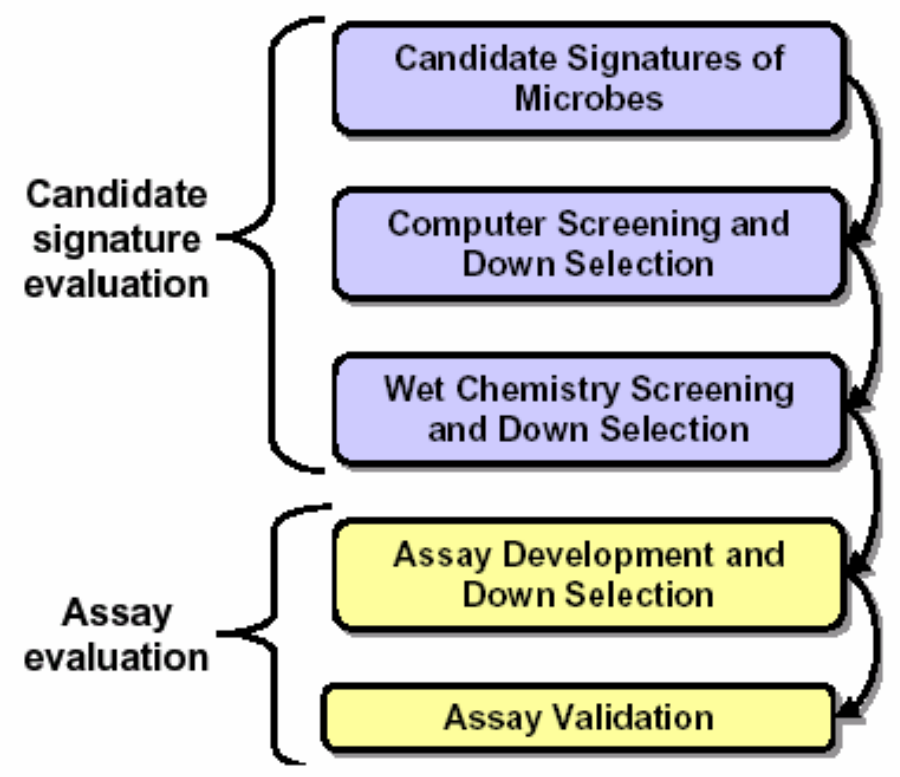

PCR with primer pairs to generate the signature fragment(s) of interest. Once candidate signatures have been identified, they are subjected to a computational screening and down-selection process. This "in silico" screening method tests the candidate regions for uniqueness when compared to all the sequence data available. The computational screening also ensures that the signature primer pairs are amenable to assay chemistry requirements and provides rapid, low-cost initial screening of signatures. The

Figure 1. The "tailored assay pipeline" process.

primers that emerge from the computational screening and down-selection are then tested against an extensive panel of DNAs and cDNAs. The bench screening consists of a panel of 2,000 to 3,000 samples, representing a wide range of organisms and backgrounds. This bench screening ensures that the primers will detect the strain diversity of the pathogen but will not react with the nucleic acids of other organisms that could be present in a sample. Primer pairs that successfully pass the wet chemistry screening criteria are advanced to the assay development stage. Assay development includes the optimization of extraction and detection protocols, so that the assays perform consistently to required specifications on the prototype equipment selected. 


\section{Bioassays and Signatures Program: Sequencing Capabilities}

The assay development process sometimes begins with sequencing a particular isolate of interest. LLNL is a founding partner to the Joint Genome Institute (JGI), who currently performs standard Sanger sequencing at its Production Genomics Facility in Walnut Creek, California. The JGI is among the world leaders in whole-genome sequencing projects devoted to microbes and microbial communities, model system vertebrates, aquatic organisms, and plants. Since 1999 they have produced $\sim 110$ billion quality bases of sequence with an

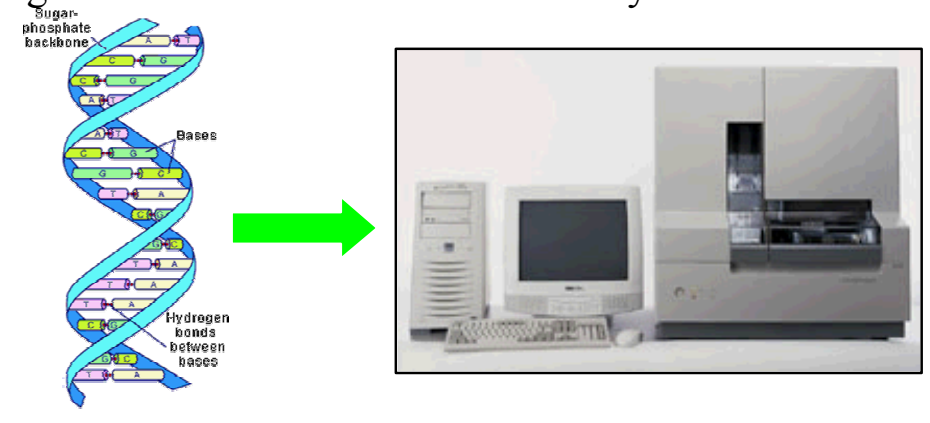
average of $92 \%$ pass rates and 655 base pair read lengths. The sequencing process for most genomes employs the whole-genome shotgun sequencing method to produce highquality draft sequence. The work begins with creation of 3-Kb, 8-Kb, and 40-Kb DNA libraries. Sequence is then obtained from both sides of the library insert, producing paired ends, typically resulting in approximately $8-9 \mathrm{X}$ depth. Reads are aligned by using various genome assemblers to produce the primary draft assembly, which consists of contigs linked into larger scaffolds by paired-end information. All genomes have at least a minimal automated annotation. For additional information see the DOE JGI Website.

The BSP also has in-house sequencing capabilities. We have developed a shotgun sequencing protocol incorporating various aspects of technology developed by Dr. Joseph DeRisi at UCSF and the JGI that is capable of generating draft sequence with 4-10X coverage of most viral genomes within 4 days. This process requires relatively pure viral nucleic acids. We have demonstrated that this level of purification can be achieved by growing virus in a matrix relatively free of contaminating host nucleic acids, virion purification by sucrose-gradient ultracentrifugation, or by using special matrices for selective purification of replicative RNA from RNA viruses

The BSP, in collaboration with CDC, USAMRIID, and USDA and the University of Texas Medical Branch has sequenced the genomes of the following human and agricultural pathogens: Ebola (3 strains), Marburg (3 strains), Sabia, Lassa (7 strains), Guanarito, Machupo (3 strains), Junin (2 strains), Rift Valley Fever (7 strains), Bluetongue, $Y$. pestis (3 strains), VESV, and West Nile virus. All genome information has been deposited into public databases (e.g., GenBank ).

Through extensive collaborations, the BSP at LLNL has unique access to over 160+ pathogen genomes that are not currently available in public web sites. Although these genomes cannot be shared with others, the BSP signature generation process can utilize this information to ensure that the resulting signatures predict to perform properly with these data. 


\section{Bioassays and Signatures Program: BioInformatics - DNA Signature development}

It is imperative to recognize the mutual interdependence of assays and bioinformatics: bioinformatics is the underpinning for assay development, providing the capacity for high throughput processing of whole genomic data to identify signatures for a variety of assay platforms. Assay development, in turn, provides feedback that drives computational enhancements and improvements. Within the BSP, bioinformatics and assays are intimately linked at each stage.

Since 2000, the bioinformatics capabilities developed under the BSP have defined the state of the art in nucleic acid assay design, with a more recent extension to the design of protein reagents. Highly automated comparative genomics and proteomics analysis systems have isolated the best regions for both nucleic acid and protein signatures.

The "KPATH" whole-genome comparative analysis software system (depicted below) first determines consensus (among one or more whole and/or draft genomes of the pathogen target) and then determines which portions of that consensus appear to be unique when compared to all other microbial genomes. Technically, a variety of anchorbased or BLAST-based algorithms are used for consensus, depending on the target genomes available and the amount of rearrangement. A suffix-tree algorithm is used for efficient computation of apparent uniqueness. KPATH mines the consensus/uniqueness result for candidate signatures based on the format desired (TaqMan ${ }^{\circ}$, nested PCR, etc.), with the entire process taking under 2 hours for the largest bacterial pathogen targets. Additional electronic checking follows to look for inexact matches that might trigger false-positives, and signature candidates are correlated with available annotation to aid in priority selection.

The KPATH DNA Signatures Pipeline database enables signature development. It is linked to provider and user entities, and supports views to details on taxonomy, signature component, melting temperature, and GC content. It also provides in silico erosion and target verification with customizable report by date, recent update, and status (true positive, false positive, false negative, indeterminate). The user can view details on percent identity, match layout, and highlight mismatches. Additional tools allow users to rapidly identify all predicted Taqman or primer pair targets from all sequenced nucleotide genomes or fragments including coordinates and gene product information.

Signature and assay maintenance is vital, as new information about pathogens and nearneighbors is being generated at ever-increasing rates. The KPATH system includes an automated "signature maintenance" process to accomplish this. New and updated microbial genomic sequence data is downloaded weekly from a variety of pertinent public sites. All fielded signatures for high-consequence pathogens are checked against this new data, with reports generated of potential false-positive and false-negative events. Over the past several years, this system has alerted us to several instances of both types 
of events, at which point we redesign the affected signatures to account for the new information.

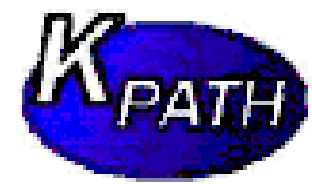

\section{Typical Tanget Pathogen For Detogtion}

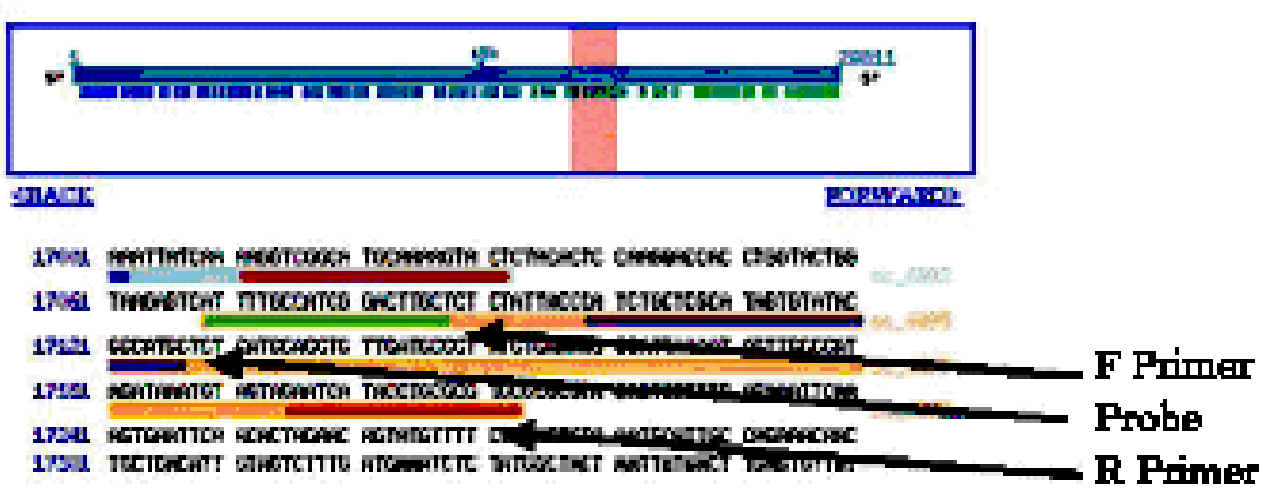

Singleplex diagnostic signatures have been transformed into multiplexed assays, however, much work remains in this area, especially as new sequence data for divergent strains of some viral targets becomes available, and multiplexed and/or degenerate signatures are essential to detect all variant strains. LLNL has developed software that effectively predicts the ability of arbitrary singleplex signatures to avoid cross-reactivity when multiplexed. Also, the standardized parameters of KPATH-derived signatures has fortuitously resulted in their working well together in multiplex format.

To enable detection of diverse target sets, we have developed software that identifies the minimal number of signatures required to capture all desired targets. This is aiding the development of reliable assays for influenza, arenaviruses, and other divergent RNA viruses. Results have been shared with various branches of CDC and other government agencies. 


\section{Bioassays and Signatures Program: BioInformatics- Protein Signature Development}

Now, LLNL extends their nucleic acid based capabilities to proteins. In FY2005 we achieved the ability to perform whole-proteome structural modeling. LLNL has built a protein structure modeling, classification, and analysis infrastructure, which has been applied to the identification of optimal protein features for targeting detection reagents. The Amino-acid to Tertiary Structure and Local-global Alignment systems provide a capability to rapidly create 3D models of proteins on a whole-proteome scale and to detect structurally conserved regions and other biologically significant fragments in protein structures. This state-of-the-art protein modeling system is coupled with highthroughput sequence- and structure-based analyses of conservation/uniqueness, and a fully automated protein annotation system, comprising LLNL's protein signature evaluation (PSE) system. PSE is the only fully integrated, high-throughput informatics infrastructure that enables a rational approach to protein detection reagent design. In addition, PSE is linked to LLNL's virulence database, which provides sequences of virulence proteins, antibiotic resistance genes, virulence islands, toxins, and select proteins that define pathogen-host interactions.

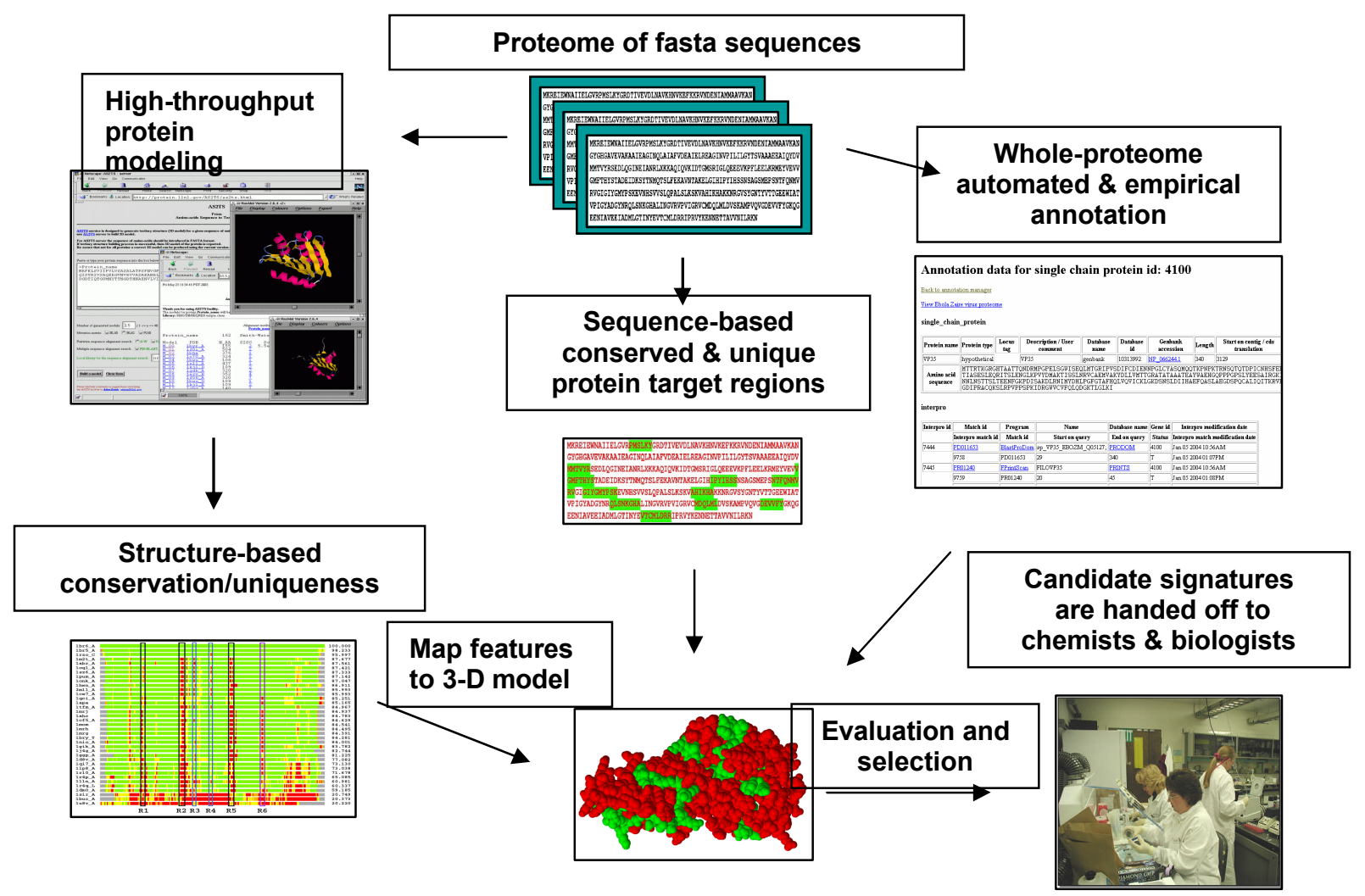

Fig. 1. High-throughput, computational protein signature evaluation system (PSE).

We have applied this computational infrastructure to discover to protein signatures for Yersinia pestis, Bacillus anthracis, variola virus, foot and mouth disease virus, ricin, and botulinum toxin. 


\section{Bioassays and Signatures Program: BioInformatics- Broad Applications}

The next generation of signatures focuses on detection of what an organism can do, not only species or strain identification. Virulence and antibiotic resistance genes often have functional homologues in many species, for which the nucleotide and even the protein sequences diverge widely. We are using Hidden Markov Models to identify diverse members of known virulence gene families and designing microarray probes to detect all sequenced variants of these genes. A virulence chip will be useful to rapidly ( $\sim 1$ day) predict what pathways, drug resistances, and toxins may be available to an uncharacterized BT or emerging pathogen, in advance of full genomic sequence data or phenotypic characterization.

The bioinformatics team leads the development of databases containing potential signatures for detection of virulence, antibiotic resistance, and engineered pathogens and toxins; containing all publicly available, organized data for virulence proteins, toxins and viral host-pathogen interaction proteins, complete with navigation tools and blast interfaces.

Support is also provided to DHS and other organizations for "scientific reachback" requests, often on short turn-around. Examples of this include a soybean rust analysis for the USDA, Yersinia analyses for USAMRIID/DHS, and Hepatitis-A assays for the FDA. A long and active collaboration with the CDC has supported their requests for help with poxvirus analyses and signatures (smallpox, vaccinia, monkeypox, cowpox, etc.), and similar analyses and signatures for SARS, influenza, brucella, Rift Valley Fever, Crimean Congo Hemorrhagic Fever, and many others.

Our computational tools have a direct impact on sequencing needs. Our sequencing analysis capability directed the USDA to sequence both the Soybean Rust fungus, and its near neighbor P.meibomiae in order to create the best diagnostics. In addition, our signature design system can run on draft and incomplete sequences, so just the fosmid sequence libraries we needed instead of the complete genomes. Without these cost savings, this achievement would not have been possible.

Several years of advanced bioinformatics research support by another federal agency has resulted in extensive SNP-based assays of published and unpublished data for organisms such as foot and mouth disease virus (FMDV) and the orthopox viruses (smallpox and related species.) These data could be readily transferred to different platforms suitable for forensic-level assays. We also have prototyped technologies suitable for massive-scale "forensic barcoding" of all select agents.

Finally, the LLNL bioinformatics team is involved in the design and use of the DHS Biodefense Knowledge Center (BKC), which uses advanced information fusion technology over a wide range of information to produce actionable intelligence. 


\section{Bioassays and Signatures: TaqMan ${ }^{\circledR}$ Nucleic Acid Assay Development}

The BSP, in collaboration with the Centers for Disease Control and Prevention (CDC) has developed, tested, optimized and validated highly specific and sensitive TaqMan ${ }^{\circledR}$ based nucleic acid assays for selected Category A and B pathogens (Figure 1). One set of these assays are deployed as part of BioWatch, and are available to the LRN for use within public health laboratories around the country and internationally. A second set of assays, designed for detection of the highest priority environmental bacterial pathogens have been operationally deployed as part of the NNSA Biological Aerosol Sentry and Information System (BASIS) laboratory and are being moved into deeply multiplexed formats for detection on next generation instrumentation. The multiplexed assay that has been developed at LLNL is capable of simultaneous detection of 8 Category A and B pathogens using a 29-plex single-tube PCR with great sensitivity and selectivity. Many additional assays for other Category A, B, and C pathogens have been developed but have not yet been validated. For example, with USAMRIID, the BSP has co-developed 53 TaqMan ${ }^{\circledR}$-based assays capable of specific and sensitive detection of a number of hemorrhagic fever viruses:: Ebola (17 signatures, providing genomic coverage across strains Reston, Sudan, and Zaire); Marburg (10 signatures capable of distinguishing between Raven and others strains of this virus); Rift Valley fever (8 signatures), Sabia (4 signatures), Lassa (7 signatures), Guanarito (4 signatures), Machupo (2 signatures), Junin (1 signature).

TaqMan PCR Assay

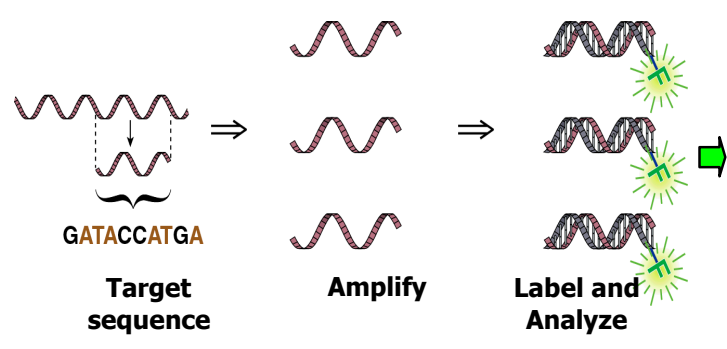

\section{TaqMan PCR Results}

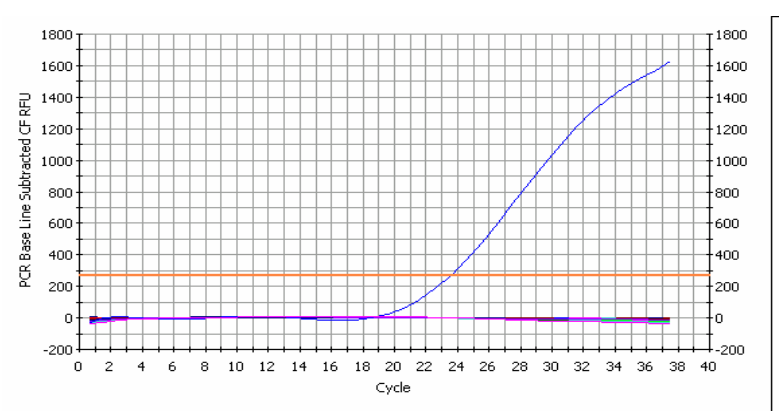

Figure 1. Conventional TaqMan ${ }^{\circledR}$ PCR process is used to amplify target regions of the genome. Typical data output is shown on the right.

BSP support to the USDA has been ongoing for two years and these efforts have generated new diagnostics for use within the National Animal Health Laboratory Network (NAHLN) and the Plum Island Animal Disease Center (PIADC). New diagnostic capabilities have been inserted into the NAHLN; these new capabilities include 34 individual TaqMan ${ }^{\circledR}$ assays. Twenty-one of these assays have been incorporated into a single-tube RT-PCR multiplexed assay capable of distinguishing between foot-and-mouth pathogens virus (FMDV) and a panel of endemic domestic and foreign animal diseases that cause clinical signs in animals that are indistinguishable from FMDV. 


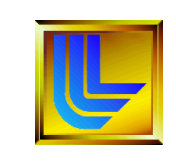

Additional work is in progress to develop and validate highly comprehensive panels of signatures and assays for emerging pathogens, including avian influenza. We are working to develop nucleic acid assays for detection of influenza. To date, 25 individual signatures have been developed and tested for the $\mathrm{H} 1, \mathrm{H} 3$. H5, and $\mathrm{H} 7$ influenza subtypes; additional work will provide signatures and assays for the remaining $\mathrm{H}$ subtypes. 


\section{Bioassays and Signatures: Multiplexed Nucleic Acid Assay Development}

Development of multiplexed assays (i.e., assays that can simultaneously test a single sample for multiple analytes) utilizes Luminex ${ }^{\circledR}$ bead-based liquid array technology. The liquid array is composed of polystyrene beads that can be individually tagged with specific antibodies, antigens, oligonucleotides, peptides, or other small molecules, enabling the development of assays that can be used for the detection of antigens, antibodies, nucleic acids, and enzymes (Figure 1). The beads are imbedded with precise ratios of red and infrared fluorescent dyes, yielding an array of 100 different beads, each with a unique spectral "address." Beads with different tags can be mixed together, and up to 100 different pathogens can be tested accurately and concurrently.

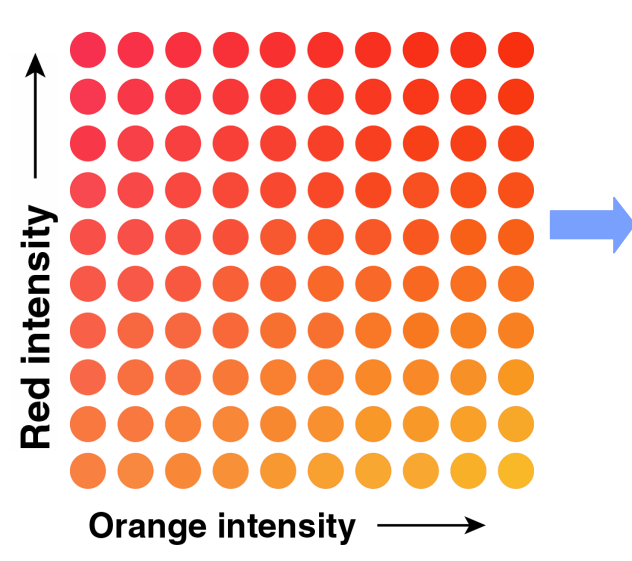

A

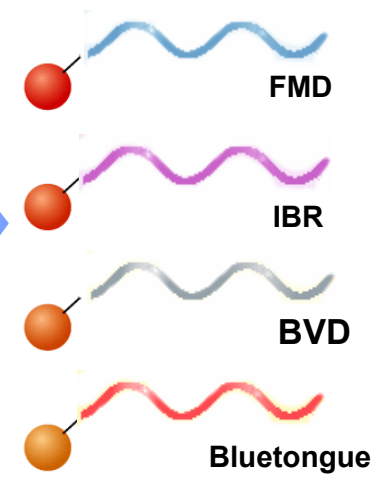

B

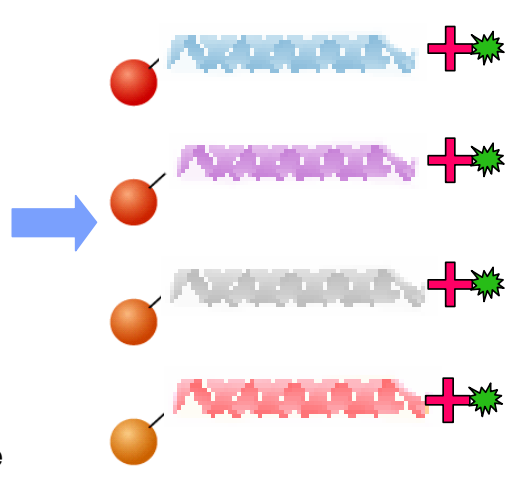

C

Figure 1. (A) 100-plex Luminex liquid array generated by intercalating varying ratios of red and infrared dyes into polystyrene latex microspheres. Each optically encoded bead has a unique spectral address. (B) Beads are coated with capture oligos. The sequence of each capture oligo matches the PCR amplicon at a site between the two primer oligos just as TaqMan probes do (C) Standard biotin-labeled PCR products from a multiplex assay are allowed to hybridize to the bead set. The fluorophore, R-phycoerythrin covalently coupled to strepavidin, is then introduced to and binds with the biotin-labeled PCR products. Beads are then individually interrogated in the flow cytometer. A red laser excites the dye molecules inside the beads, and a green laser excites the fluorescent molecules bound to the bead surfaces.

The BSP and CDC co-developed a deeply multiplexed multi-loci panel of nucleic acid assays (27-plex) for detection of 8 different biological threat agents; this panel of assays is under final evaluation by the CDC and will be validated in the LRN using a multicenter collaborative study in March, 2006. Validated assays will be transitioned to the private sector for commercialization and dissemination within the LRN. These assays have also been developed and optimized in a TaqMan ${ }^{\circledR}$ format and transitioned to the CDC. This multiplexed panel represents the next-generation of BioWatch assays: the panel may eventually replace the TaqMan ${ }^{\circledR}$-based primary and confirmatory detection assays currently used by the LRN BioWatch laboratories. Using the multiplexed panel offers the BioWatch program 4 significant advantages over the current methods:

1. Major reduction in reagent and labor costs. Because the multiplexed assays are contained within a single tube and are performed simultaneously, the multiplexed 
panel offers significantly enhanced detection capabilities with more than an $90 \%$ reduction in costs and significant savings in labor relative to the costs and time required to run each individual assay.

2. The multiplexed panel comprises both the primary and confirmatory testing in a single test. This will eliminate the time required to run the confirmation panel following a screen reactive; this will result in a confirmed positive hours sooner than under current protocols.

3. The multiplexed nucleic acid panel employs the same detection/analysis equipment as the previously-validated multiplexed immunoassays: therefore, the LRN labs will have the ability to test positive samples using an orthogonal detection method, and, the LRN labs will have toxin-detection capabilities, all available on the same instrument platform.

4. These assays are compatible with high-throughput sample "surge" processing and analysis capacity robotics: sample surge capability was fully-developed and tested in 2004, and will be piloted in the AgDDAP later this year. Our sample processing capacity currently stands at about 4,000 samples per 10 hour shift, using a single line of equipment.

Working with the USDA, the BSP has developed a deeply multiplexed (21-plex) assay for detection of 8 different foreign and domestic vesicular disease viruses. These assays can be used to differentiate foot-and-mouth disease virus from other foreign and domestic vesicular disease viruses, where all the viruses cause clinical signs in animals that look alike. This panel was demonstrated in an inter-laboratory comparison study in the National Animal Health Laboratory Network (NAHLN); and will be evaluated with clinical diagnostic samples over the course of the next year. The multiplexed assay panel will enter validation in FY2006, and LLNL is actively seeking partners for commercialization of this panel. 


\section{Bioassays and Signatures: Multiplexed Immunoassay Development}

Like the multiplexed nucleic acid panels described above, the BSP has employed the liquid array technology to develop deeply multiplexed panels of antibody based assays. These antigen detection assays (immunoassays) employ a typical sandwich immunoassay format where antigen-specific capture antibodies are immobilized on the polystyrene beads, antigen is introduced and allowed to bind the beads, and the bound analyte is subsequently detected using secondary antibodies indirectly labeled with the fluorescent reporter, phycoerythrin (PE).

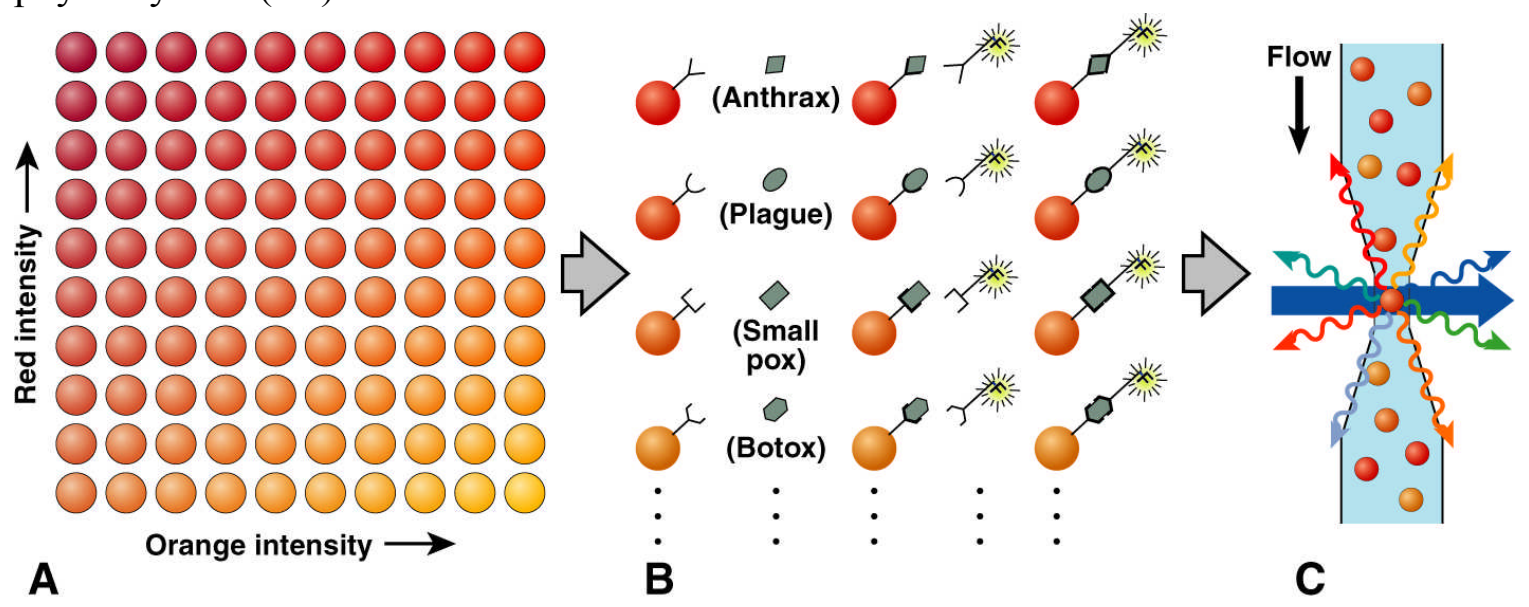

Figure 1. A) 100-plex Luminex liquid array generated by intercalating varying ratios of red and infrared dyes into polystyrene latex microbeads. Each optically encoded bead has a unique spectral address. B) Beads are coated with capture antibodies specific for target antigens. After incubation with the antigens, secondary or detector antibodies are added, followed by addition of the fluorescent reporter, phycoerythrin to complete the "antigen sandwich". C) The beads are analyzed in the flow cytometer. Beads are interrogated one at a time. A red laser classifies the bead, identifying the bead type. A green laser quantifies the assay on the bead surface - only those beads with a complete sandwich will fluoresce in the green, and the signal is a function of antigen concentration.

A multiplexed panel of immunoassays for detection of biothreat agents has already been developed, validated and disseminated to the LRN; this panel is currently being used to augment the detection capabilities of BioWatch laboratories, by adding protein toxin detection capabilities. This panel employs a 9-plex, although many additional assays have been developed since this panel was validated. The BSP also retains a fullydeveloped panel of multiplexed immunoassays for detection of simulants of biological threat agents. These assays may find broad utility among the private sector or others who are developing biodetection instrumentation, where the integrated systems can be tested and optimized at the benchtop under Biosafety Level 1 or 2 conditions. The stimulant panel enables high-quality testing and optimization, prior to testing of integrated systems involving BSL-3 level agents. 
The BSP has developed a panel of multiplexed immunoassays for the detection of common respiratory pathogens, including influenza $A$ and $B$, parainfluenza (types 1-3), $\mathrm{RSV}$, and adenovirus. The performance of this panel is under evaluation in a clinical study, an dearly results indicate sensitivity and specificity on par with other antibodybased methods, but improvements are being made. The BSP has also combined the respiratory panel (12-plex) with the validated biothreat agent panel (9-plex), and we have demonstrated that these two panels can be effectively combined with no loss in sensitivity or specificity to any individual assay. Thus, the panel may have utility as a tool for surveillance or syndromic screening.

The liquid array platform is exceptionally versatile and can be used to develop assays for a number of analytes. In addition to the antigen capture assays, we have employed the converse, where the objective is to capture antibodies from solution. Here, the beads are covalently coupled to protein, and used to capture antibodies specific to those proteins from solution. In collaboration with the Canadian National Center for Foreign Animal Diseases, we have also developed a multiplexed protein-based assay for the differentiation of naturally-infected versus vaccinated animals (DIVA) for foot-andmouth disease. These assays, also employing the liquid array technology, will be evaluated at the international level (OIE); if the assays meet evaluation criteria, they may help to resolve the issue of "vaccinate to live" vs. "vaccinate to kill" that has plagued the international FMDV community for more than 30 years. Following validation, they will be transitioned to commercial diagnostic company. 


\section{Bioassays and Signatures: Development of Protein Based Detection Reagents}

Pathogen specific 3-dimensional protein features are a rich but largely untapped resource for affinity-based detection. There has been a significant gap in the need for robust affinity reagents targeting pathogen protein signatures and the ability to produce these reagents. Driven by these needs, LLNL BSP and others have established a "protein pipeline" reagent development effort. The effort is based on identifying unique protein signatures with bioinformatic-driven computational modeling coupled with high efficiency reagent discovery capabilities based on reagent molecular platforms that are stable, modifiable and manufacturable. The resulting computationally-focused high throughput reagent production is focused on generating sets of reagents that meet the high confidence levels required of the DHS end users and can be mass produced by a commercial vendor for pennies per reaction, upon demand, without relying on the use of animals or animal cell lines.

The Protein Pipeline project has identified unique protein signatures for five threat agents, Bacillus anthracis, Yersinia pestis, ricin, botulinum toxin, and Variola major., including multiple proteins from the whole organism threat agents. The methods used to generate these initial signature sets are general and are currently being applied to provide a steady-state of highvalue threat agent protein targets for reagent development. The target sets can be used to inform and focus virtually any type of reagent discovery technology from combinatorial to rational design approaches.

The Protein Pipeline project has focused on evaluating and

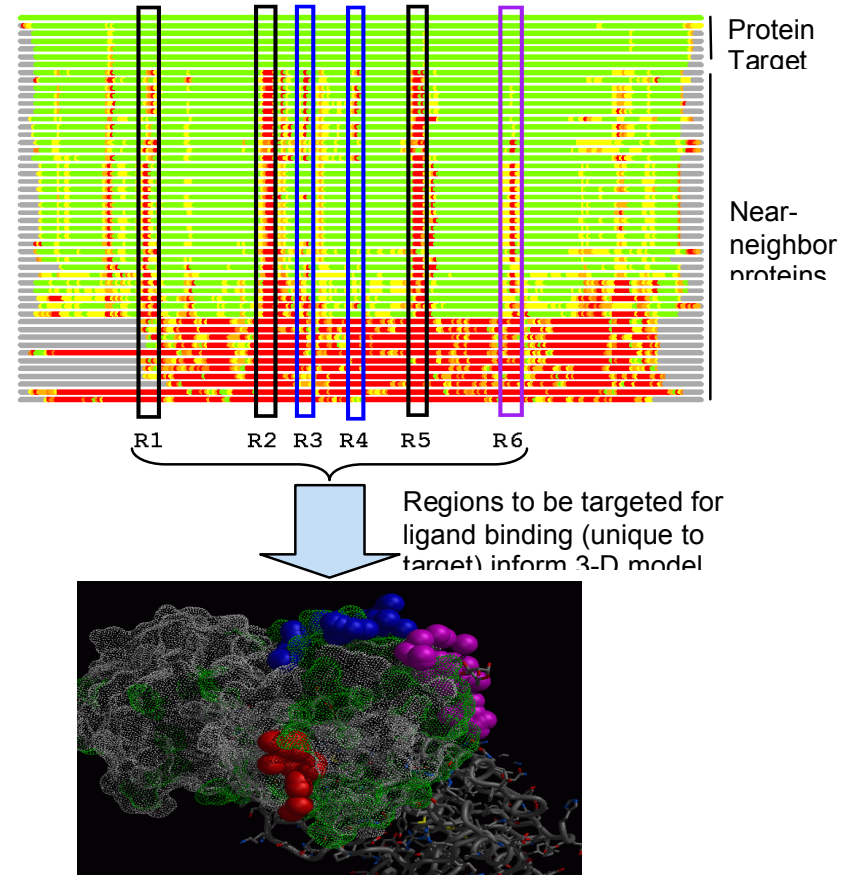

employing complimentary reagent discovery technologies that together provide the highest chance of success to develop affinity reagents for virtually any pathogen protein target. We have given high priority to technologies and molecular scaffolds that are robust, broadly applicable, and efficient. Currently, potential protein affinity reagents have been identified, tested for binding to their protein target, and are currently being assessed for their ability to bind to the organism/ intact toxin. The initial evaluation of the first new protein-signature based detection reagents will be completed this spring. 


\section{Bioassays and Signatures: Functional Assays and Signatures}

Post September $11^{\text {th }}$ and the anthrax letters of Fall 2001, a shift in the direction of biodefense research has emerged with a new urgency for basic, discovery-driven science to provide novel solutions for biodefense preparedness. Basic science now has a clear role in supporting critical translational applications for Homeland Security. For example, host-pathogen interactions (Figure 1), which reflect the balance of host defenses and pathogen virulence mechanisms, contain key signatures or biomarkers that can be useful for unveiling virulence characteristics of pathogens, for detecting emergent or engineered agents, and for presymptomatic and presyndromic surveillance of infectious diseases. LLNL groups focus on virulence mechanisms and host-pathogen interactions of Yersinia pestis and Bacillus anthracis, the etiological agents of plague and anthrax, as well as other viral,

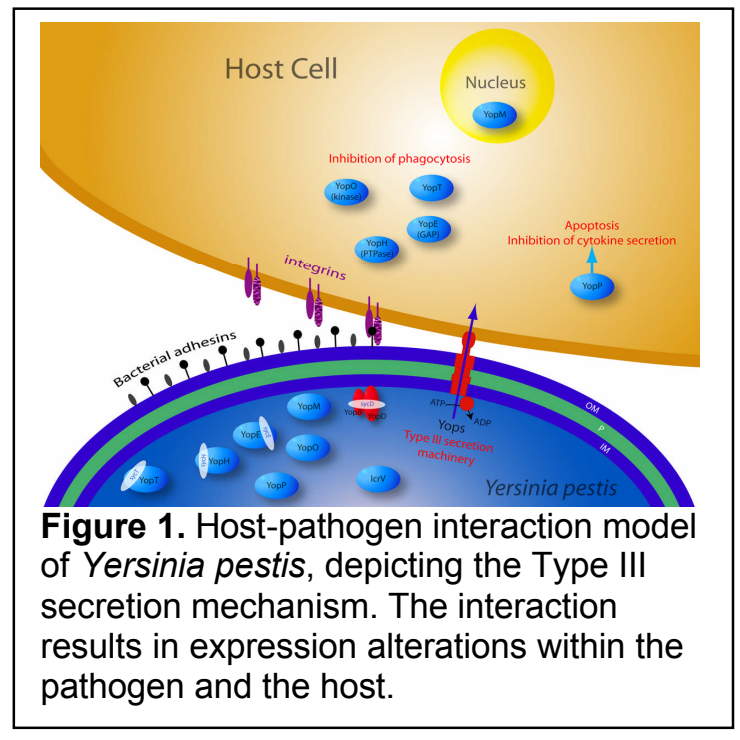
microbial and toxin treats to the civilian, agricultural and food sectors.

To address the need for orthogonal detection assays that can provide a level of functional or threat characterization, we have studied the proteomic, genomic and phenotypic traits within diverse microbial pathogens as well as proteomic, genomic, clinical, and immunohistopathological categorization of host response to diverse pathogens in rodent, cell, and whole blood models. From our studies, we are developing the basis for understanding pathogen diversity as it relates to effective detection. For example, different strains of $Y$. pestis and B. anthracis exhibit differential expression and metabolic

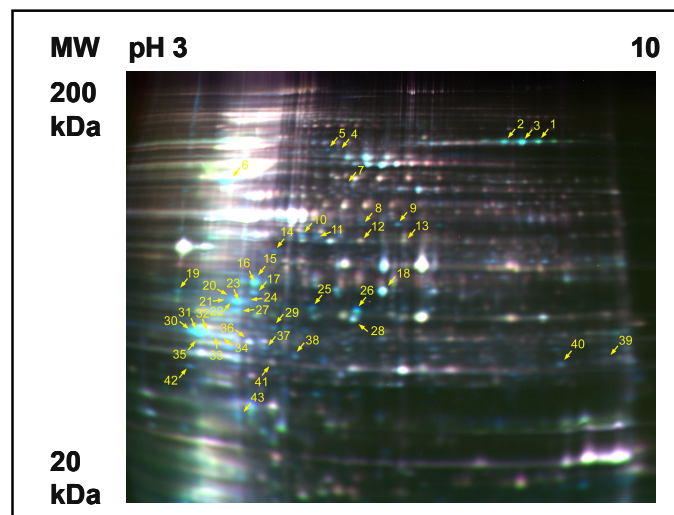

Figure 2. Y. pestis proteomic map showing differentially expression detected by multiplexed fluorescent-based 2-D DIGE, identified by mass spectrometry. phenotype that correlate with diversity in degree of virulence. This knowledge has identified candidate signatures for environmental monitoring. In the case of $Y$. pestis, it is known that alteration in physiological growth conditions from the temperature and calcium concentration found in the flea vector to that found in the mammalian host can induce virulencerelated expression in $Y$. pestis. In our studies, hundreds of proteins were differentially expressed between these growth conditions, and more notably between strains with known differences in virulence level (Figure 2). 


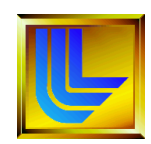

Hierarchical clustering of proteomic data has enabled us to distinguish diverse pathogens based on rapid proteomic screening with comparison to a "Pathogen Signature Library". For example, Figure 3 shows extended data analysis of differential expression within 4 diverse strains. From this data, it is possible to blindly classify unknown samples. Additional metabolic data from phenotypic arrays provided evidence of biochemical pathways not indicated by the genome annotation or known biochemistry of $Y$. pestis. Further, we determined that phenotype could distinguish same species strains. Significant differences were detected between virulence inducing and vector-like growth conditions, and between same species strains with diverse virulence levels. We have also linked phenotype and functional metabolic pathway information to virulence factor expression and host response, providing a novel and unprecedented approach to threat characterization via a Systems Biology view.

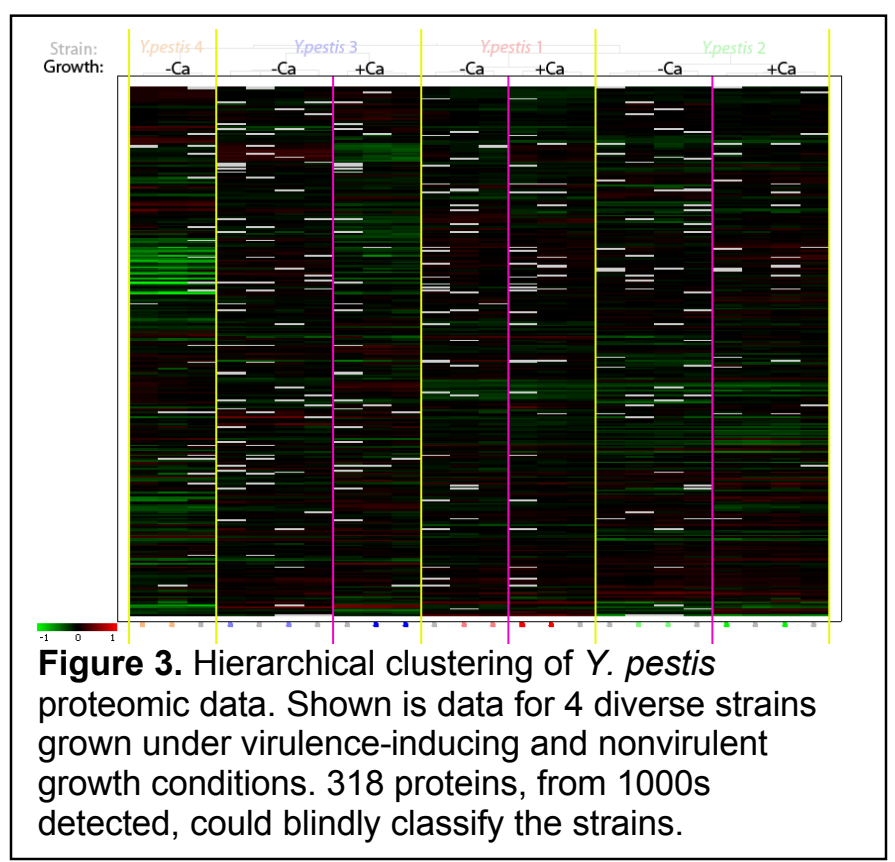

Similarly, from host response characterization it is possible to distinguish exposure to multiple pathogens including: virulent from avirulent pathogens; $Y$. pestis from $B$. anthracis; near neighbors from each other; and, most strikingly, two virulent clinical isolates of $Y$. pestis with differences in virulence level. Distinct cytokine profiles shown in Figure 4 suggest that

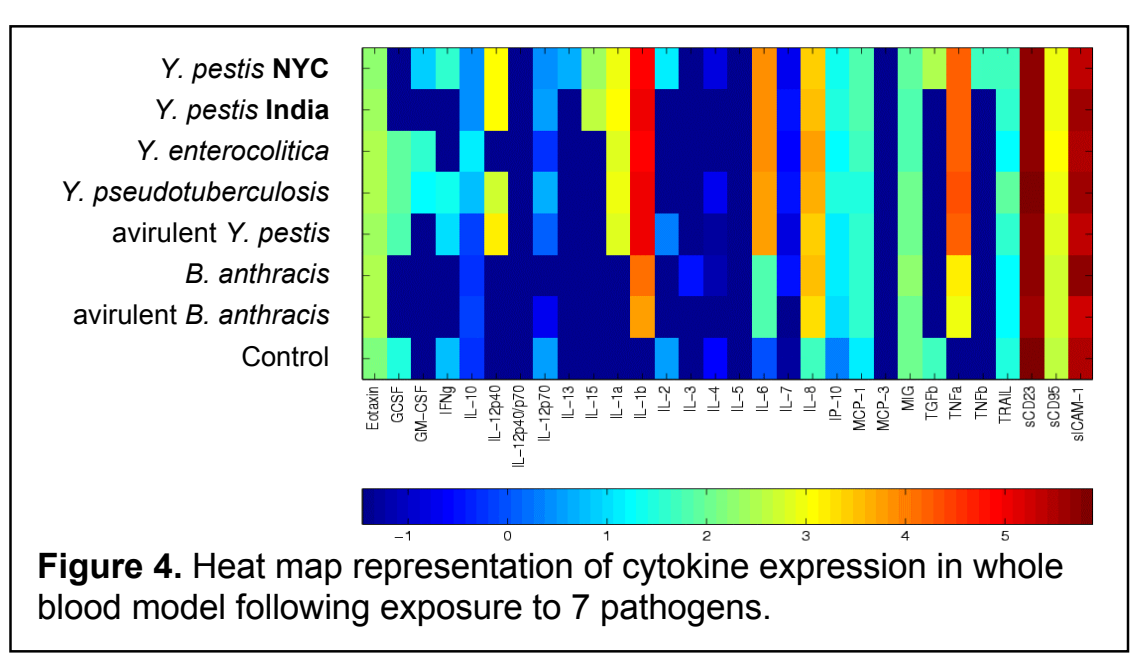

host-response signatures can be used for rapid rule-out diagnosis of anthrax, plague and other diseases. These results represent an obvious translational application to detection and surveillance with the added feature of threat assessment, such as would be needed following a pathogen release or an infectious

disease outbreak. From a detection perspective, our ultimate vision is that of a dipstick, mouthswab, or breathalyzer type of diagnostic for infectious diseases and pathogen identification/characterization based on signatures from host-pathogen interactions studies. 


\section{BioAssays and Signatures: High Throughput Laboratory}

The high-throughput laboratory (HTL) has been developed, maintained, and housed at LLNL and is a certified BioSafety Level Two Laboratory (BSL-2). The HTL is a semiautomated laboratory that uses robotic liquid handling platforms and 96-well assay technologies to rapidly process incoming samples. We have demonstrated the ability to process more than 4,000 samples from end-to-end using just 2 technicians and a single line of equipment in a 10-hour shift. Protocols have been developed such that the system can accept and process many different types of samples, including swabs, swipes, filters, and liquids, comprising different environmental sample matrices including soil, fresh water, and aerosols from a variety of filter matrices.

The HTL has been successfully deployed many times, and can be operationally relocated and functioning within 24 hours. This capability offers sample "surge" capacity that could be used to respond to outbreaks or suspected events of bioterrorism, or to support/augment existing laboratory capabilities such as those found within the public health community. 


\section{Bioassays and Signatures: Dissemination of Assay Products into User Communities}

Over the past two years, the BSP has devised a well-developed strategy for testing, validation, commercialization, and final dissemination of TaqMan and Multiplexed assay products into user communities. We have developed well organized and detailed Assay Report Cards that capture in seven main tabs all of the information necessary to determine the capabilities and limitations of any nucleic acid assay. These tabs include:

1. Bioinformatics (all target and near-neighbor sequences used to generate signatures; type of target [DNA/RNA; virus, bacterium, fungus]; genome size)

2. Signature summary (sequences of all primers and probes used in the final assay; GC content; length; melting temperature; location on genome; target gene description)

3. Level of discrimination (eg. Species, strain, subspecies, etc.)

4. List of all samples use for screening (including all soils, zoos, microbes, aerosols, targets, and near-neighbors)

5. Erosion report (detailed info. on potential cross-reactivities)

6. TaqMan report (including Limit of Detection on multiple targets)

7. Multiplex assay report (number of signatures included in Multiplex assay, crossreactivities, limits of detection, etc.)

These report cards have gone a long way to bridge the gaps that exist between research and translation of assays into the hands of the user community. The BSP at LLNL comprises unparalleled assay development capabilities, a unique and comprehensive diagnostics testbed that enables assays and/or diagnostic platforms to be thoroughly evaluated by directly comparing performance to other state-of-the-art assays/instrumentation, and a training and technology transfer program that has placed assays, instrumentation, and training directly into the hands of our partners at the CDC/LRN and the United States Department of Agriculture National Animal Health Laboratory Network [USDA/NAHLN]. 


\section{BioInstrumentation Development Program: Bioassays and Signatures: A Multiplexed Diagnostic Platform for use at Point-of-Care}

The preceding pages have provided a description of our multiplexed assay development efforts, using both nucleic acid (PCR) and protein (immunoassay) methods. We have also developed similar panels for the detection of common respiratory pathogens; these panels are finding utility in the clinical setting, especially at point-of-care. The nucleic acidbased assay panel is under development, but currently provides for rapid, sensitive, and specific detection of up to eight different respiratory pathogens simultaneously from a single sample. Our multiplexed assays have been developed for FluID $_{\mathrm{x}}$, an integrated system designed for use at point-of-care. FluID ${ }_{x}$ is capable of sample preparation and processing (e.g., nucleic acid extraction), performing highly multiplexed real-time PCR nucleic-acid based assays, data analysis and reporting, and system decontamination between samples, where all functions are completely automated. FluID ${ }_{x}$ currently employs an 18-plex panel, comprising assays for 8 respiratory pathogens (Influenza Virus A and B subtypes, Parainfluenza Virus Types 1-3, Respiratory Syncytial Virus, Adenovirus, and SARS) where each agent is represented by multiple loci, and 5 unique internal controls. Results on a patient sample can be provided in about 3 hours.

The performance of FluID ${ }_{x}$ will be benchmarked in a year-long pilot, beginning October, 2005. An instrument has been deployed into the hospital emergency room at the University of California Davis Medical Center in Sacramento, CA. The pilot will enable training, demonstration, and evaluation of the system by hospital personnel The prototype system is depicted in the photograph in Figure 1a; shown in Figure 1b is an artist's rendering of the instrument repackaged into a clinically-relevant outer housing.

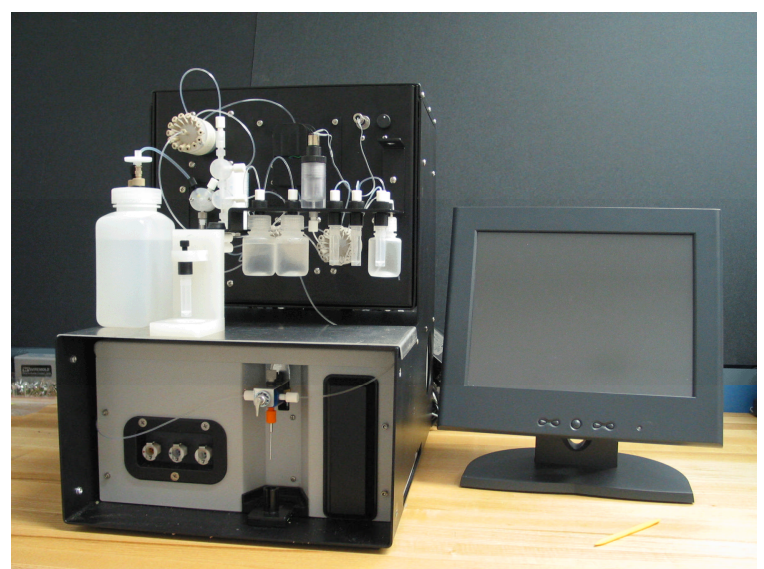

(A)

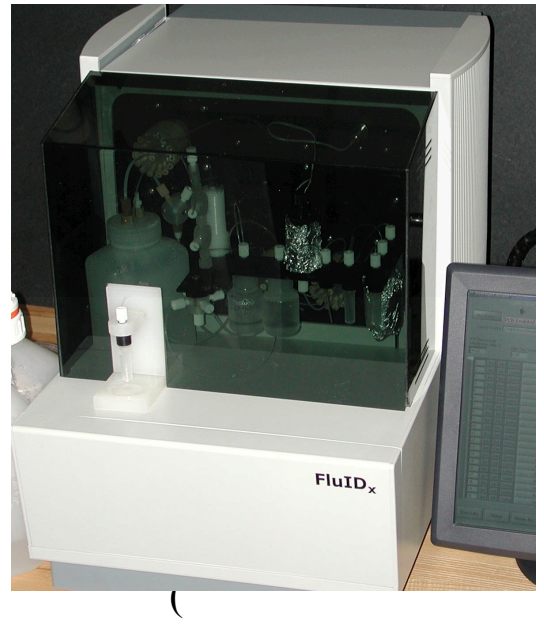

B)

Figure 1. (A) Photograph of the prototype device, showing monitor, fluidics manifold and Luminex 50 detection system. (B) FluID device with housing. 


\section{BioInstrumentation Development Program: An Advancement in Detection Systems - BioBriefcase}

The CBNP and Sandia National Laboratory have collaborated to produce the BioBriefcase, a briefcase-sized, multiplex, autonomous, broad-spectrum bioagent detector. This instrument monitors the environment and provide "detect-to- treat" capability for civilian applications, relevant to the Bioagent Autonomous Networked Detectors (BAND) provided by DHS. We have developed a concept that uses microfluidics and chip-based modules for performing sample preparation and analysis. This device employs three separate and orthogonal analysis methods - PCR, immunoassay, and protein signature - to provide two analysis methods each for the detection of bacteria and viruses (PCR and immunoassay) and toxins (immunoassay and protein signature). All three of the analysis modes employ capillary electrophoresis with laser-induced fluorescence detection. The BioBriefcase features multiplex capability, dramatically reduced reagent consumption, decreased cost, and decreased size, while maintaining or improving sensitivity and response time compared to currently available technologies. The Bio Briefcase was successfully field tested with simulants in early FY06. 


\section{BioInstrumentation Development Program: High Throughput Rapid Viability Analysis}

Current innovative biodetection technologies are often limited by issues of front-end sample processing, and are challenged to efficiently transfer pathogen cells or spores from environmental or clinical samples to a form compatible with the small volumes required for nucleic acid or immuno-based assays. The method limit of detection is negatively impacted by losses during this transfer process as well as by the assay's inability to measure pathogen in the entire sample. In addition, methods typically do not distinguish viable and potentially infectious pathogen from dead pathogen, important information for determining the risk of exposure. Current methods for extraction and analysis of viable pathogens are labor and time-intensive, with low throughput, and have relatively high detection limits. Although traditional real-time PCR assays provide rapid, specific and sensitive detection of pathogens, they fall short of determining viability. Recently, a Rapid Viability (RV)-PCR method based on a significant change in real-time PCR response after incubation of the sample has been developed and tested on several biothreat (BT) agents at LLNL. The method was able to detect growth of B. anthracis within 4 hours from a complex environmental sample (HEPA vacuum sock filled with debris from a mailroom) containing contamination below detectible levels (40 spores/mL). Substantial time reductions were achievable for viability determination for other BT organisms (Table 1).

Table 1. Comparison of Rapid Viability PCR with conventional culture-based analysis ${ }^{\mathrm{a}}$.

\begin{tabular}{|l|l|l|}
\hline Criterion $^{\prime}$ & Rapid Viability PCR $^{\mathbf{b}}$ & Conventional Assay $^{\mathbf{c}}$ \\
\hline Analysis time for: & & \\
B. anthracis & $4-6 \mathrm{hr}$ & $2-4$ days \\
Y. pestis & $6-8 \mathrm{hr}$ & $3-5$ days \\
Brucella sp. & $6-8 \mathrm{hr}$ & $5-7$ days \\
F. tularensis & $8-10 \mathrm{hr}$ & 7 days \\
\hline Sample processing & 200 dirty wipes $/ 4-6 \mathrm{hr}$ for & 30 wipes $/ 8 \mathrm{hr}$ for $B . a .$, \\
throughput/day & 1 person and 1 robot & 2 people \\
\hline Detection limit & $1-10$ spores per wipe & NA \\
\hline
\end{tabular}

a The cost/analysis for RV-PCR is approximately one-half that of the conventional assay due to decreased labor requirements. b PCR analysis at time 0 was below detection. The change in PCR response at the endpoint showed an increase of at least 2 logs in cell population; $\mathrm{c} N \mathrm{NA}=$ data not available.

In parallel, high throughput sample processing protocols that are compatible with PCR analysis have been developed, allowing processing of hundreds of samples (e.g., wipes, filters, filtrates) in short turn around times (4-6 hr) using commercially available robotics. In field-testing, the protocols could accommodate a large amount of background debris without decreasing throughput and detection limits were consistently $\leq 10$ spores. Testing also showed that 10 live $B$. anthracis surrogate spores could be detected in a background or $10^{6}$ chlorine dioxide-killed spores. Both automated and field-deployable protocols significantly reduce the amount of manual sample handling $g$, therefore reducing the risk of exposure to personnel. Other features include the ability to archive the culture and lysates for additional PCR and biochemical analyses and validation. The protocols are 
compatible with all relevant sample types including fluids, filters, wipes, swabs, and vacuum socks. Air samples from wet collection methods such as cyclone samplers reported to maintain viability of collected microorganisms could easily be incorporated into sample processing protocols. Modifications are being made to high throughput protocols to accommodate vegetative cell targets, maintaining cell viability throughout the process allowing the technology to be widely applied to bacterial pathogens.

QuickTime $^{\mathrm{TM}}$ and a 